\title{
Silicon chips detect intracellular pressure changes in living cells
}

Rodrigo Gómez-Martínez ${ }^{1}$, Alberto M. Hernández-Pinto ${ }^{2}$, Marta Duch ${ }^{1}$, Patricia Vázquez ${ }^{2}$, Kirill Zinoviev ${ }^{1}$, Enrique J. de la Rosa ${ }^{2}$, Jaume Esteve ${ }^{1}$, Teresa Suárez ${ }^{2}$, José A. Plaza ${ }^{1 *}$

${ }^{1}$ Instituto de Microelectrónica de Barcelona, IMB-CNM (CSIC), Campus UAB, 08193, Cerdanyola, Barcelona, Spain.

${ }^{2}$ Centro de Investigaciones Biológicas, CIB (CSIC), C/Ramiro de Maeztu 9, 28040, Madrid, Spain.

The ability to measure pressure changes inside different components of a living cell is important because it offers an alternative way to study fundamental processes that involve cell deformation ${ }^{1}$. Most current techniques such as pipette aspiration ${ }^{2}$, optical interferometry ${ }^{3}$, or external pressure probes ${ }^{4}$ use either indirect measurement methods or approaches that can damage the cell membrane. Here we show that a silicon chip small enough to be internalized into a living cell can be used to detect pressure changes inside the cell. The chip, which consists of two membranes separated by a vacuum gap constituting a Fabry-Pérot resonator, detects pressure changes that can be quantified through the intensity of the reflected light. Using this chip, we show that extracellular hydrostatic pressure is transmitted into HeLa cells and that these cells can endure hypoosmotic stress without significantly increasing their intracellular hydrostatic pressure. 
Scientific interest in the intersection of micro- and nanotechnologies with biology is focused on providing new tools to study fundamental questions in cell biology ${ }^{5,6,7}$. Fabrication based on these techniques offers the potential to develop integrated devices with nanosized moving parts $^{8}$ and allows for new opportunities for mechanical analysis of cells ${ }^{1,9,10}$. However, their use has been focused on extracellular or invasive techniques $^{11}$. Conversely, micro- and nanoparticles can be internalized inside living cells and have been used in numerous studies in cell biology. In addition, silicon-based particles have revealed their superiority in biological imaging and drug delivery because of their inherent biocompatibility ${ }^{12,13}$. Recently, we demonstrated a fabrication technique based on semiconductor technologies of silicon microparticles for single-cell labelling ${ }^{14,15}$. Using chemical functionalisation, we also proved that they could react with the intracellular medium ${ }^{16}$.

Existing techniques for the indirect measuring of intracellular pressure include methods which induce a large deformation of the cell by aspiration ${ }^{2}$, or methods which detect cell-volume variations ${ }^{1,3}$. Conversely, the servo-null technique allows for a direct measurement by inserting a micropipette as a pressure probe ${ }^{4}$; however, the cell membrane is mechanically damaged. Thus, the measurement of extracellular loads transmitted inside the cell, and particularly to a subcellular component, has not been directly demonstrated. The cell is a highly complex and practically unexplored mechanical system where the membranes, the cytoskeleton and the extracellular matrix provide its structural integrity. 
Here, we fabricated a nanomechanical chip which can be internalized to detect intracellular pressure changes in living cells and allows an interrogation method based on confocal laser scanning microscopy (CLSM).

The design consisted of a mechanical sensor (Fig. 1a) defined by two membranes separated by a vacuum gap and an optical reference area. The membranes acted as parallel reflecting mirrors constituting a Fabry-Pérot resonator that is partially transparent for some wavelengths ${ }^{17}$. External pressure, $P$, deflected the membranes and changed the gap, $t_{\text {gap }}$ (Fig. 1b). Hence, the intensity of the reflected light at the centre of the membranes, $I_{r_{-} \text {Centre, }}$ for a given wavelength, $\lambda$, is modulated by the $P$. The reference area is used for focusing purposes. Briefly, the sensing principle is based on the acquisition of images for a given $\lambda$ and the quantification of the $I_{r_{-} C e n t r e}$.

The fabrication processes included the deposition of three structural and three sacrificial layers, poly-silicon and silicon oxide, respectively (Fig. 1c and Supplementary Fig. 1). Polycrystalline silicon was selected as the structural material because of its elastic behaviour and high reliability ${ }^{18}$. The lateral dimensions of the mechanical membranes were fixed to $3 \mu \mathrm{m} \times 3 \mu \mathrm{m}$ (Fig. 1d). Analytical and simulated analyses showed that a mechanical deformation was highly dependent on the membrane thickness and the linear response versus $P$ (Fig. 1e and Supplementary Fig. 2). Thus, we selected 50-nm thick membranes to achieve a theoretical mechanical sensitivity of $5.5 \mathrm{~nm} / \mathrm{bar}$. The high refraction index of poly-silicon provides a spectral selectivity of the structure and, subsequently, a high sensitivity to $P$. Theoretically, the optical reflection of the structure (Fig. 1f) showed a resonance valley that was a function of $t_{\text {gap }}$ and $\lambda$ (Fig. 1g, h). Thus, $P$ shifted the reflection curve towards smaller values $\left(\sim 2 \times \Delta t_{\text {gap }}\right)$; for $\lambda$ fixed high variations of the reflection could be obtained. Finally, $t_{\text {gap }} \sim 300 \mathrm{~nm}$ was selected upon considering the high optical sensitivity and cell internalization capabilities. 
The fabricated devices were validated using a bright-field optical microscope (BFOM). The experiment showed a minimum reflection for $\lambda \sim 570 \mathrm{~nm}$ (Fig. 2a). Fixed $\lambda$, the $I_{r_{-} \text {Centre }}$ increased versus $P$ for a $\lambda>580 \mathrm{~nm}$, and it decreased for a $\lambda<560 \mathrm{~nm}$. CLSM images with superior resolution allowed to develop an image-processing algorithm to detect the pressure loads based on a quantification of the mean intensities of three regions of interest (Supplementary Figs. 3, 4 and 5). External pressure was applied from 0 to 1 bar and from 1 to 0 bar. The $I_{r_{-} S e n s o r}$ noticeably decreased by the $514-\mathrm{nm}$ and increased by the 594-nm laser wavelengths (Fig. 2b).

To test the sensor inside living cells, we took advantage of our previous experience of internalizing silicon microparticles inside HeLa cells by lipofection ${ }^{16}$. Sensors were easily localised by optical light microscopy because of the higher reflectivity of polysilicon, while CLSM showed the specific location of the chip in the cytoplasm (Fig. 3a, b). Sensors only represent the $0.2 \%$ of the total volume of a typical HeLa cell (Supplementary Fig. 6). After transfection, a proportion of HeLa cells in the culture displayed vacuoles due to the lipofection procedure. Our experiments showed that these vacuoles did not affect cell fitness nor viability (Fig. 3a, b and Supplementary Movie S1) and disappeared when cells were back to normal culture conditions (Supplementary Fig. 7). Sensor-containing HeLa cells, with or without vacuoles, divided normally (Fig. 3c and Supplementary Movie S2), displayed active mitochondria (Supplementary Fig. 7) and were healthy 9 days later (Supplementary Fig. 8). On the other hand, we confirmed that the vacuoles $\mathrm{pH}$ oscillates between 4-6 and that the device was not degraded inside HeLa cells 9 days after the lipofection (Supplementary Fig. 8). This result was in good agreement with the non-degradation of polysilicon in solutions buffered at different $\mathrm{pH}$ between 4-9 (Supplementary Fig. 8). 
We next analysed the mechanical transmission of extracellular pressure to a subcellular component. The presence of the sensor inside a vacuole had several inherent advantages. First, it can give information of how an external pressure is mechanically transmitted to the organelles. Second, it prevents eventual induction of a mechanical cross-sensitivity on the device by other organelles or cytoskeletal filaments, which can induce small forces and displacements (Supplementary Fig. 2). Third, better-quality CLSM images are obtained when the sensors are immersed in a medium of a uniform refractive index (Supplementary Fig. 9). Figure 4a shows overlaid images of transmitted light and laser channels in which the vacuole and different parts of the device can be easily recognised. External pressure was applied from 0 to 1 bar and from 1 to 0 bar. A comparison between the $I_{r_{-} \text {Sensor }}$ inside the vacuole and the calibrated sensor in air showed close proportional changes (Figs. $4 b$ and $2 b$ ) and confirmed that the extracellular pressure is transmitted into the vacuole (Supplementary Fig. 10). Figure 4c results demonstrated the capability of detecting pressure fluctuations inside a cell. The reflection from the sensor depends on the optical properties of the surrounding media, however the position of the resonance is almost invariant (Supplementary Fig. 11). We also observed that the $I_{r_{-} \text {Sensor }}$ was reversible, which showed that the pressure inside the vacuole followed the extracellular pressure changes. This result discarded possible cross-sensitivities (Supplementary Figs. 4 and 12).

We then analysed the effect of the exposure of HeLa cells to an osmotic shock $(1 / 10$ water dilution of the standard cell medium). A new batch of chips was fabricated for this study showing a minimum reflection of the spectrum at $490 \mathrm{~nm}$. The induced osmotic pressure predicted by van't Hoff's law is expected to produce a hydrostatic pressure of $\sim 7$ bars inside the cell. Thus, the predicted osmotic shock pressure should shift $\sim 42 \mathrm{~nm}$ the Fabry-Pérot resonator minimum reflection. Chips located both in a 
subcellular compartment (vacuole) (Supplementary Fig. 13) and in the cytosol of HeLa cells showed that reflection profiles of chips inside cells before and after the osmotic shock were practically the same (Supplementary Fig. 14). The extrapolated wavelength for the minimum reflection was very similar in all the cases (Fig. 4d). We could infer that the pressure change inside the cell should be below few hundreds millibars. Thus, our results provide direct evidence of low intracellular hydrostatic pressure when HeLa cells are submitted to a great osmotic stress.

Extracellular pressure is a common load in many real cases; human cells experience $\Delta P$ $=0.2$ bar from feet to head, which can be higher during human activity, and deep sea animals can be exposed to 200 bar upon diving ${ }^{19}$. Hard-wire tensegrity models postulate that the cytoskeleton can resist mechanical forces ${ }^{20}$. Our experiments support that the cytoskeleton of human HeLa cells do not mechanically withstand extracellular pressures in the studied range and under our experimental cell-culture conditions. Thus, extracellular pressure is transmitted through the cytosol to the inner compartments. The implication would be that intracellular transmission of fluid-pressure follows the Pascal's law. Conversely, our data also show that intracellular pressure remains practically unaltered inside cytosol and vacuoles during an osmotic shock, supporting that these cells are preventing inward flow of water across the membrane ${ }^{21}$. Typically, when animal cells endure an osmotic shock, they adapt and do not dramatically increase intracellular pressure ${ }^{22-24}$.

Additional work remains to increase the device's sensitivity for accurate pressure measurements, including thinner mechanical layers, autofocus and tilt-stage systems and computer-assisted measurements. Mechanical forces are not very well understood and are involved in basic cellular processes, such as cell migration ${ }^{25,26}$, diseases $^{27-29}$ and development ${ }^{30}$. Intracellular mechanical sensors will provide inside information on 
these cellular forces which will open new opportunities. We believe that this is a first step towards a broad field of intracellular nanochips that will provide a different perspective on fundamental problems in cell biology.

\section{Methods.}

\section{Imaging acquisition during pressure experiments.}

BFOM. Experiments were performed with an Eclipse ME600 upright optical microscope (Nikon). A 100x magnification by a 0.8 NA long-distance objective LU Plan ELWD 3.5 (Nikon) was used. Images were recorded via an 8-bit colour CCD camera (DXM1200F; Nikon) using the advanced control software Nikon ACT-1 (Automatic Camera Tamer). Band-pass filters (THORLABS, Ltd) coupled with a YMNCB11 filter slider (Nikon) were used to select the wavelength of the incident light. CLSM. Confocal images were acquired with a confocal Leica TCS-SP5 microscope (Leica Microsystems $\mathrm{GMbH}$ ) using the $514 \mathrm{~nm}, 561 \mathrm{~nm}$ and $594 \mathrm{~nm}$ excitation laser wavelengths $(\mathrm{AOTF}=1 \%)$ for the first batch of fabricated chips, and the $458 \mathrm{~nm}, 476$ $\mathrm{nm}, 488 \mathrm{~nm}, 496 \mathrm{~nm}$ and $514 \mathrm{~nm}$ excitation laser wavelengths $($ AOTF $=1 \%)$, for the second batch of fabricated chips. The confocal analysis was conducted in the AOBS reflection mode, with 16 bit-depth resolution and in the X-Y-Z scan mode. A $63 x / 0.9$ HCX APO water objective (Leica Microsystems $\mathrm{GMbH}$ ) was used. The image acquisition time is $\sim 25 \mathrm{~s}$. The images were pre-analysed by LAS AF software (Leica Microsystems GMbH).

Cell manipulation and osmotic shock. Chips were lipofected inside human HeLa cells by a protocol previously described by us [16]. HeLa cells were incubated $12-16 \mathrm{~h}$ in the 
lipofection medium. Cell viability was analysed by incubating cells with Cell Tracker Green and MitoTracker Red (Molecular Probes, Invitrogen, USA) for $15 \mathrm{~min}$ at $37^{\circ} \mathrm{C}$. HeLa cells were fixed with 4\% paraformaldehyde in PBS for $45 \mathrm{~min}$. The nuclei were stained with DAPI (MolecularProbes, USA) and the cells were mounted with Fluoromont-G (Southern Biotech, Alabama,USA) for microscopy. HeLa cells were also incubated with Calcein AM, MitoTracker Red, DiOC and Lysosensor Red (Molecular Probes, Invitrogen, USA), for direct observation under the CLSM following the manufacturer's recommendations. Cells were grown on glass coverslips and observed under the CLSM inside a live-imaging Ludin chamber. To expose cells to an osmotic shock, standard DMEM medium with 10\% Foetal Bovine Serum (Molecular Probes, Invitrogen, USA) was $10 \%$ diluted in deionized water and was perfused in the Ludin chamber.

Cell viability imaging. Cells were observed under a TCS SP2 AOBS CLSM with 63 X oil immersion lens (Leica Microsystems GMbH, Germany). Green fluorescence was monitored with excitation and emission settings of $488 \mathrm{~nm}$ and 505-550 nm, respectively. Red fluorescence was monitored with excitation and emission settings of $561 \mathrm{~nm}$ and $580-610 \mathrm{~nm}$, respectively. A 351-nm laser line was used to image nuclei and fluorescence emission was measured at 415-460 $\mathrm{nm}$. Chips were imaged with a 488-nm laser line and they were detected by reflected light at 480-495 $\mathrm{nm}$. Time-lapse microscopy was performed with a Leica AF6000 LX model DMI6000B and pictures were taken every 10 min. HeLa cells videos were processed with Leica imaging software. 
Statistical analysis. Data analysis was performed with Graph Pad Prism 4 software. ANOVA and Bonferroni test were used to compare intra-group data (chip inside cell or chip in air data sets). On the other hand, $\chi^{2}$ test was used to compare pressure data from calibration chips in air versus chips inside cells.

Extrapolate $\lambda$ for minimum reflection. $\lambda$ for the minimum reflection (Fig. $4 \mathrm{~d}$ ) were extrapolated from data (Supplementary Figure 14) by adjusting the mean intensities for the 5 selected-lasers to a second order polynomial. The minimum corresponded to the $\lambda$ where the first derivative of the function was zero.

\section{References.}

1. Stewart, M.P. et al. Hydrostatic pressure and the actomyosin cortex drive mitotic cell rounding. Nature 469, 226-230 (2011).

2. Rand, R.P. \& Burton, A.C. Mechanical properties of red cell membrane .i. Membrane stiffness + intracellular pressure. Biophys. J. 4, 115-135 (1964).

3. Strohmeier, R. \& Bereiterhahn, J. Hydrostatic-pressure in epidermal-cells is dependent on ca-mediated contractions. J. Cell Sci. 88, 631-640 (1987).

4. Kelly, S.M. \& Macklem, P.T. Direct measurement of intracellular pressure. Am. J. Physiol. 260, C652-C657 (1991).

5. Whitesides, G.M. The 'right' size in nanobiotechnology. Nature Biotechnol. 21, 1161-1165 (2003). 
6. Singhal, R. et al. Multifunctional carbon-nanotube cellular endoscopes. Nature Nanotechnology 6, 57-63 (2011).

7. Tian, B. Z. et al. Three-dimensional, flexible nanoscale field-effect transistors as localized bioprobes. Science 329, 830-834 (2010).

8. Arlett, J.L., Myers, E.B., \& Roukes, M.L. Comparative advantages of mechanical biosensors. Nature Nanotechnol. 6, 203-215 (2011).

9. Cross, S.E., Jin, Y.S., Rao, J. \& Gimzewski, J.K. Nanomechanical analysis of cells from cancer patients. Nature Nanotechnology 2, 780-783 (2007).

10. Balaban, N.Q. et al. Force and focal adhesion assembly: a close relationship studied using elastic micropatterned substrates. Nat. Cell Biol. 3, 466-472 (2001).

11. Vaziri, A. \& Gopinath, A. Cell and biomolecular mechanics in silico. Nature Materials 7, 15-23 (2008).

12. Fan, J.Y. \& Chu, P.K. Group IV Nanoparticles: Synthesis, Properties, and Biological Applications. Small 6, 2080-2098 (2010).

13. Tasciotti, E. et al. Mesoporous silicon particles as a multistage delivery system for imaging and therapeutic applications. Nature Nanotechnology 3, 151-157 (2008).

14. Fernandez-Rosas, E. et al. Intracellular polysilicon barcodes for cell tracking. Small 5, 2433-2439 (2009).

15. Novo, S. et al. A novel embryo identification system by direct tagging of mouse embryos using silicon-based barcodes. Human Reproduction 26, 96-105 (2011).

16. Gomez-Martinez, R. et al. Intracellular silicon chips in living cells. Small 6, 499-502 (2010). 
17. Born, M. \& Wolf, E. Principles of optics, $6^{\text {th }}$ Eds., (Pergamon Press, Oxford 1980).

18. French, P.J. Polysilicon: a versatile material for microsystems. Sensors and Actuators A 99, 3-12 (2002).

19. Myers, K.A., Rattner, J.B., Shrive, N.G. \& Hart, D.A. Hydrostatic pressure sensation in cells: integration into the tensegrity model. Biochemistry and Cell Biology 85, 543-551 (2007).

20. Ingber, D.E. Tensegrity I. Cell structure and hierarchical systems biology. $J$. Cell Sci. 116, 1157-1173 (2003).

21. Borgnia, M., Nielsen, S., Engle A. \& Agre, P. Cellular and Molecular Biology of the Aquaporin Water Channels. Annu. Rev. Biochem. 68, 425-458 (1999).

22. Spagnoli, C., Beyder, A., Besch, S. \& Sachs, F. Atomic force microscopy analysis of cell volume regulation. Physical Review E. 78, 031916 (2008)

23. Finan, J.D. and Guilak, F. The effects of osmotic stress on the structure and function of the cell nucleus. J. Cell Biochem. 15, 109(3), 460-7 (2010).

24. Pietuch, A, Brückner, B.R, and Janshoff, A. Membrane tension homeostasis of epithelial cells through surface area regulation in response to osmotic stress. BBA Mol. Cell Res. 1833 (3), 712-722 (2013).

25. Tambe, D. T. et al. Collective cell guidance by cooperative intercellular forces. Nature Materials 10, 469-475 (2011).

26. Trepat, X. et al. Physical forces during collective cell migration. Nature Physics 5, 426-430 (2009). 
27. DuFort, C.C., Paszek, M.J. \& Weaver, V.M. Balancing forces: architectural control of mechanotransduction. Nature Rev. Mol. Cell Biol. 12, 308-318 (2011).

28. Jaalouk, D.E. \& Lammerding, J. Mechanotransduction gone awry. Nature Rev. Mol. Cell Biol. 10, 63-73 (2009).

29. Fritsch, A. et al. Are biomechanical changes necessary for tumour progression? Nature Physics 6, 730-732 (2010).

30. Wozniak, M.A. \& Chen, C.S. Mechanotransduction in development: a growing role for contractility. Nature Rev. Mol. Cell Biol. 10, 34-43 (2009).

Acknowledgments. This work was supported by the Spanish projects TEC2009-07687E and TEC2011-29140-C03-01. P.V. was supported by Centro de Investigación Biomédica en Red de Diabetes y Enfermedades Metabólicas Asociadas-Instituto de Salud Carlos III (CIBERDEM-ISCIII). We thank M. Calvo of Centros Científicos y Tecnológicos-Universidad de Barcelona (CCiT-UB), M.T. Seisdedos (CIB), J. Monteagudo (Leica Microsystems S.L.) and D. Megias of Unidad de Microscopía Confocal-Centro Nacional de Investigaciones Oncológicas (CMU-CNIO) for their CLSM experimental assistance and A. Bosch (CCiT-UB) for her assistance with the image processing. We also thank the clean-room staff of IMB-CNM for the fabrication of the chips. 
Author Contributions All authors discussed the results and contributed to writing the manuscript. M.D., R.G-M. and J.E. conceived and guided the chip fabrication. The optical design and analysis was carried out by K.Z. The biological experiments were performed by A.M.H.P. and P.V., designed by A.M.H.P. and E.J.d.l.R and planned and coordinated by T.S.. R.G-M. performed the experimental characterisation of the chips and the data analysis. J.A.P. conceived and directed the project.

\section{Additional Information}

Supplementary information accompanies this paper at www.nature.com/naturenanotechnology. Reprints and permission information is available online at http://npg.nature.com/reprintsandpermissions/. Correspondence and requests for materials should be addressed to J.A.P. The authors have no competing financial interests. 


\section{Figure Captions}

\section{Figure 1| The design and sensing principle of the chip.}

a, The schematics of the chip. b, The working principle. The intensity of the incident light, $I(\lambda)$, and the intensities of the reflected light from the reference area, $I_{r_{-}} \operatorname{Ref}(\lambda)$, and from the centre of the membrane, $I_{r_{-} \text {Centre }}(\lambda, P)$. (Insets) Mechanical deformation of the membranes versus $P$. c, The schematics of the chip fabrication at two stages. (Top) A poly-silicon layer Polyl defined the bottom membrane; two sacrificial silicon oxide layers, $O x 3$ and $O x 2$, defined the Fabry-Pérot cavity; and two poly-silicon layers, Poly2 and (Bottom) Poly3, defined the top membrane. The Poly $1+$ Poly $2+$ Poly 3 patterning delimited the device. The devices were released by the etching of the silicon oxide layer Oxl (not shown). d, A SEM image of the fabricated device. Scale bar $=3 \mu \mathrm{m}$. e, The theoretical displacement of the membrane, Displ $l_{m e m b}$, versus the membrane thickness, $t_{m e m b}$, and (Inset) versus $P$. f, A schematic view of the optical multilayer structure defining the Fabry-Pérot resonator (refractive index, $n$; layer thickness, $t$ ). The simulated results of the reflection as a function (g) of $\lambda$ and (h) $t_{\text {gap }}$ (medium, $50-\mathrm{nm}$ thick poly-silicon layer, vacuum gap, 50-nm thick poly-silicon layer, medium).

\section{Figure 2| The validation of the sensing principle.}

a, BFOM experiment in air medium. (Top) Experimental true-colour images taken by an 8-bit colour CCD camera versus $\lambda$ and $P$. Band-pass filters from 500 to $650 \mathrm{~nm}$ were used to select the working $\lambda$. (Bottom) Normalized $I_{r_{-} C e n t r e} / I_{r_{-}} R e f \quad$ (255 a.u. for $\lambda=500$ $\mathrm{nm}), P$ induces a lateral displacement of the curve towards a smaller $\lambda$. Fixed $\lambda$, positive or negative sensitivities are observed (black arrows). Error bars, $\pm 10 \%$ (based on

measurement uncertainty from images) b, CLSM experiment in air medium. The $I_{r_{-}}$Sensor 
versus $P$ from 16-bit images. Lasers $\lambda=514 \mathrm{~nm}, 561 \mathrm{~nm}$ and $594 \mathrm{~nm}$ were used to select the working $\lambda$. Positive and negative sensitivities are also observed for $\lambda=594 \mathrm{~nm}$ and $\lambda=514 \mathrm{~nm}$ respectively. $I_{r_{-} \text {Sensor }}$ decreased for $0 \leq P \leq 0.75$ bar and increased for $P$ $=1$ bar $\lambda=561 \mathrm{~nm}$ as it is close to resonance valley of Fabry-Pérot spectrum. Error bars, $\pm 5 \%, 9 \%$ and $8 \%$ for $\lambda=514 \mathrm{~nm}, 561 \mathrm{~nm}$ and $594 \mathrm{~nm}$ respectively (based on measurement uncertainty from images).

\section{Figure 3| The silicon chips inside human cells.}

A HeLa cell displaying an internalized chip (white arrow) inside a vacuole (a) and inside the cytoplasm (b). The cells were loaded with vital dyes CellTracker Green and MitoTracker Red before fixation. (a, b) include (Top-left panel) a transmitted visible light image, (Top-right panel) an overlay of confocal images and (Bottom) an orthogonal projection of confocal images showing that the chip is inside the cell. c, A HeLa cell containing a device inside the vacuole can proceed through mitosis (individual frames taken from Supplementary Movie S2; the time format is hh:mm). Scale bar $=10 \mu \mathrm{m}$.

\section{Figure 4| The detection of pressure changes inside cells.}

a, False-colour 16-bit CLSM images of HeLa cells with a chip inside a vacuole (white arrow). (Left to right) Images show the cells under transmitted visible light and the reflected light of selected lasers $(\lambda=514,561$ and $594 \mathrm{~nm})$. Insets show the chip inside a vacuole. White scale bar $=20 \mu \mathrm{m}$. (Insets) Black scale bar $=5 \mu \mathrm{m}$. b, The $I_{r_{-} \text {Sensor }}$ versus $P$. Error bars, $\pm 5 \%, 9 \%$ and $8 \%$ for $\lambda=514 \mathrm{~nm}, 561 \mathrm{~nm}$ and $594 \mathrm{~nm}$ respectively (based on measurement uncertainty from images) c, A comparison between the $I_{r_{-}}$Sensor 
$(\lambda=594 \mathrm{~nm}) / I_{r_{-} \text {Sensor }}(\lambda=514 \mathrm{~nm})$ ratio versus $P$ for chips in air and inside cells. Statistical analysis ${ }^{*} \mathrm{p}<0.05 ; * * \mathrm{p}<0.01 ; * * * \mathrm{p}<0.001$ (ANOVA - Bonferroni test). No significant differences were found between the chips performance in air and inside cells $\left(\chi^{2}\right.$ test $\left.\mathrm{p}=0.6922\right)$. Data are presented as the mean \pm SEM of five independent observations. d, Extrapolated $\lambda$ for the minimum reflection of chips in the cytosol and inside vacuole before and after the osmotic shock showing non-significant shift of the reflected spectrum after the shock. 

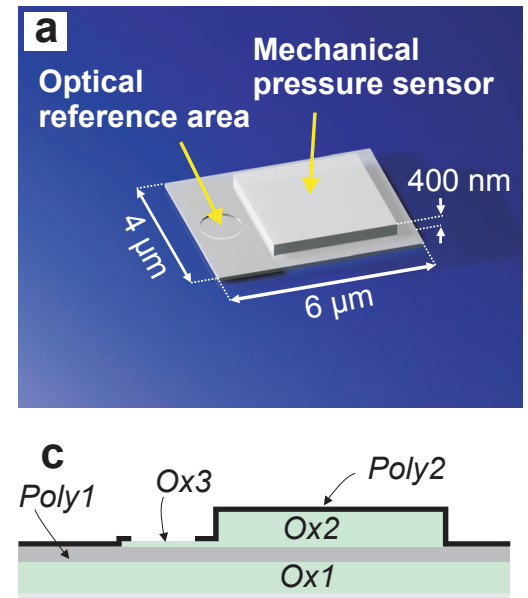

Si substrate

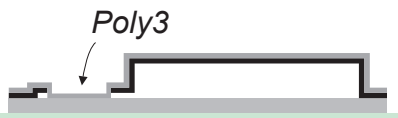

f

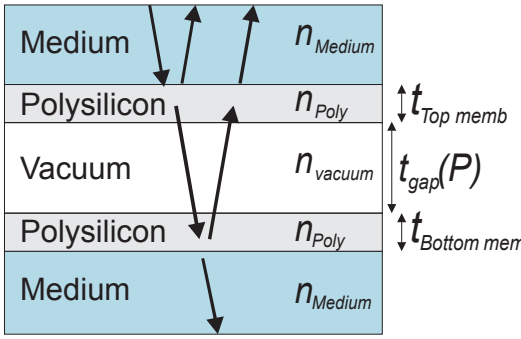

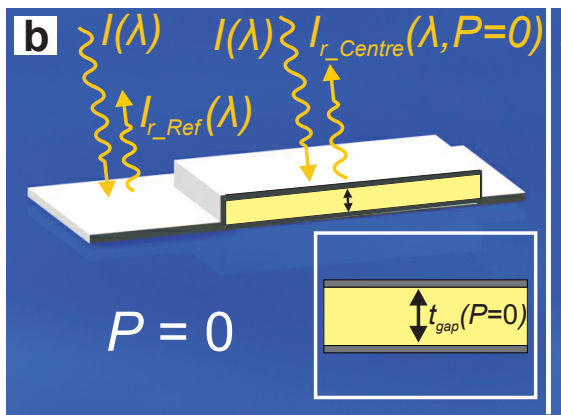
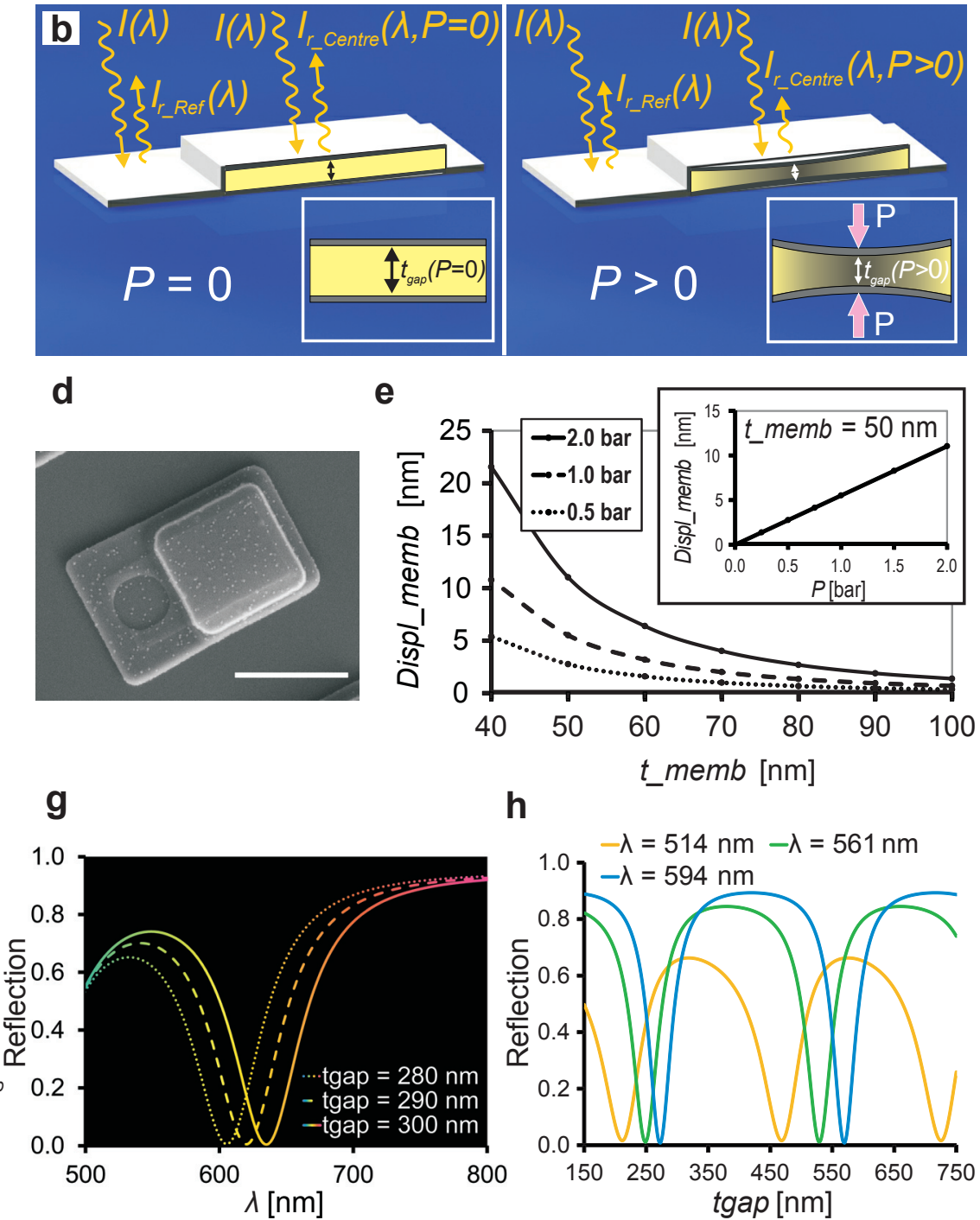

h

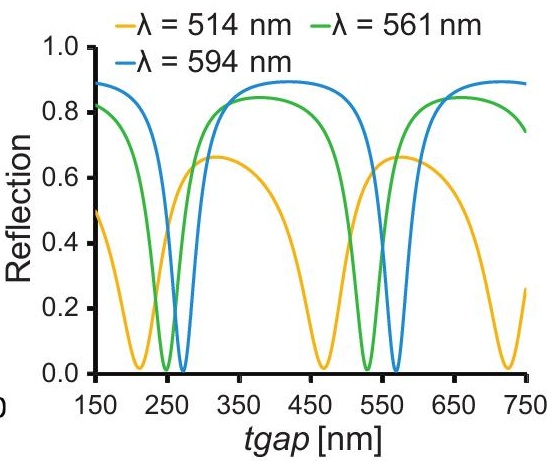



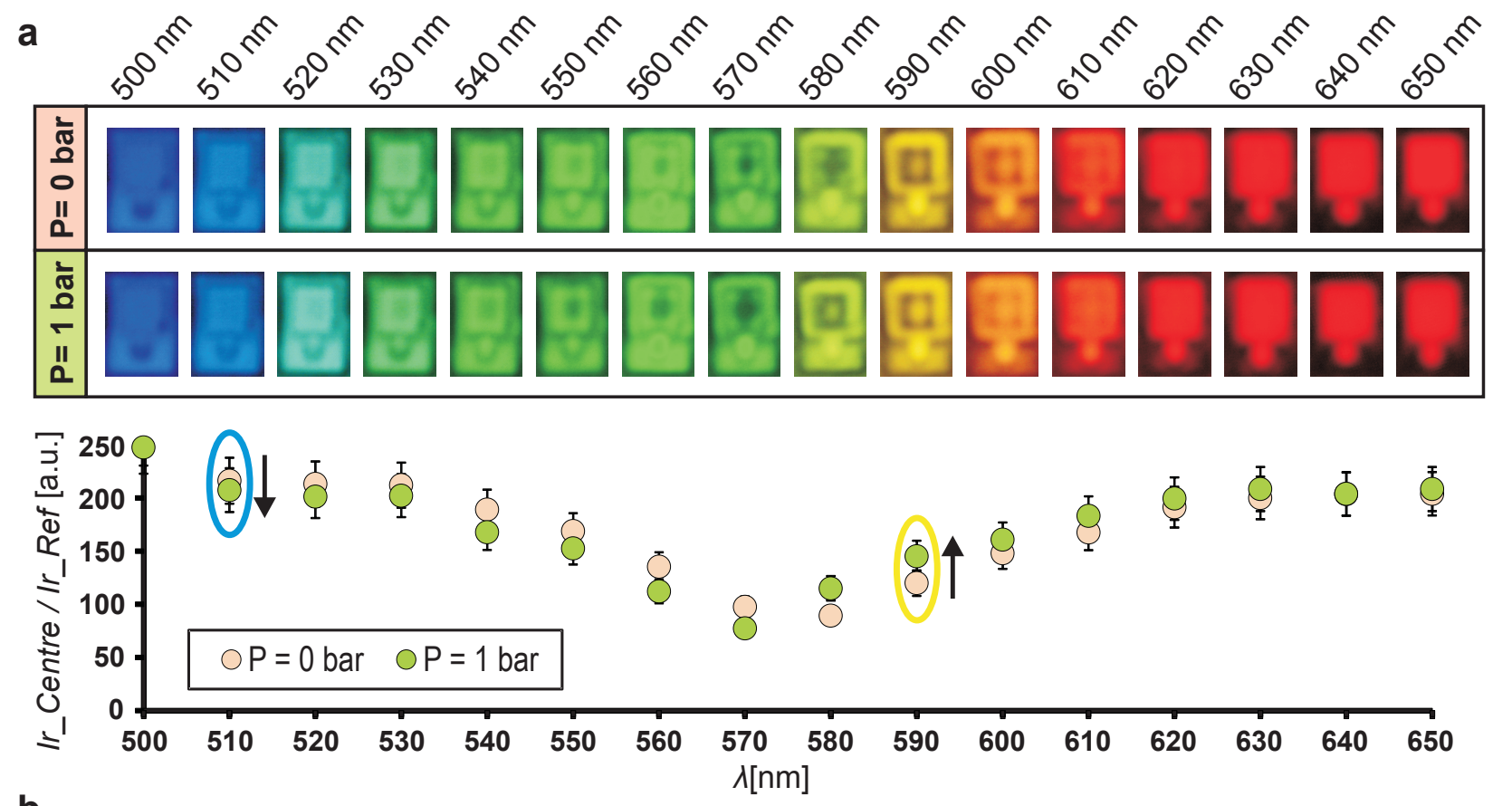

b
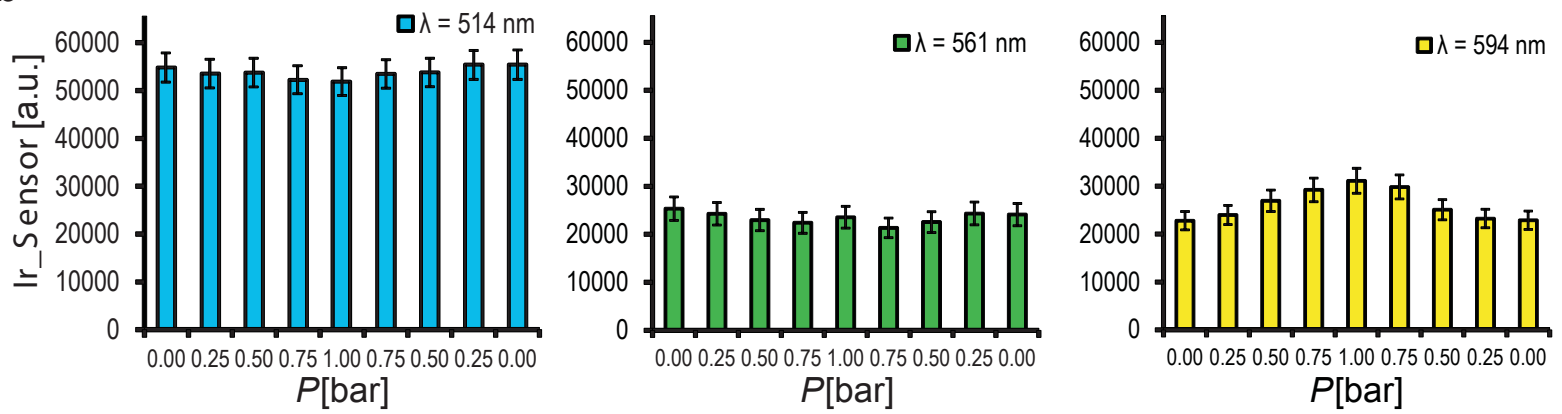


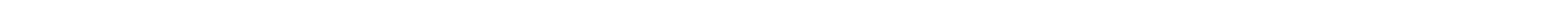


